\title{
Why Clowns Taste Funny: The Relationship between Humor and Semantic Ambiguity
}

\author{
Tristan A. Bekinschtein, ${ }^{1}$ Matthew H. Davis, ${ }^{1}$ Jennifer M. Rodd, ${ }^{2}$ and Adrian M. Owen ${ }^{1,3}$ \\ ${ }^{1}$ Medical Research Council Cognition and Brain Sciences Unit, CB2 7EF Cambridge, United Kingdom, ${ }^{2}$ Cognitive, Perceptual and Brain Sciences Research \\ Department, University College, London WC1N 3BG, United Kingdom, and ${ }^{3}$ Centre for Brain and Mind, University of Western Ontario, London, Ontario, \\ Canada N6A 5B7
}

What makes us laugh? One crucial component of many jokes is the disambiguation of words with multiple meanings. In this functional MRI study of normal participants, the neural mechanisms that underlie our experience of getting a joke that depends on the resolution of semantically ambiguous words were explored. Jokes that contained ambiguous words were compared with sentences that contained ambiguous words but were not funny, as well as to matched verbal jokes that did not depend on semantic ambiguity. The results confirm that both the left inferior temporal gyrus and left inferior frontal gyrus are involved in processing the semantic aspects of language comprehension, while a more widespread network that includes both of these regions and the temporoparietal junction bilaterally is involved in processing humorous verbal jokes when compared with matched nonhumorous material. In addition, hearing jokes was associated with increased activity in a network of subcortical regions, including the amygdala, the ventral striatum, and the midbrain, that have been implicated in experiencing positive reward. Moreover, activity in these regions correlated with the subjective ratings of funniness of the presented material. These results allow a more precise account of how the neural and cognitive processes that are involved in ambiguity resolution contribute to the appreciation of jokes that depend on semantic ambiguity.

\section{Introduction}

What makes us laugh? This is an age old question, but how humor is instantiated in the brain has only recently lent itself to neuroscientific investigation (Goel and Dolan, 2001). Recent functional neuroimaging studies have demonstrated that verbal jokes (Goel and Dolan, 2001), humorous TV shows (Moran et al., 2004), and visual gags (Gallagher et al., 2000; Marjoram et al., 2006, Wild et al., 2006; Samson et al., 2009) all increase activity in the left inferior frontal gyrus (IFG) and the posterior temporal lobe.

One crucial component of many jokes is the disambiguation of words with multiple meanings (Attardo, 2001; Goel and Dolan, 2001). For example, in the joke "Why don't cannibals eat clowns? Because they taste funny!", the punchline depends on two meanings of the word "funny" (amusing and odd/bad). Both meanings are appropriate in this joke. Thus, "Why don't cannibals eat rotten eggs? Because they taste funny!", is not humorous,

Received Sept. 27, 2010; revised May 16, 2011; accepted May 19, 2011

Author contributions: T.A.B., M.H.D., J.M.R., and A.M.O. designed research; T.A.B. performed research; J.M.R. contributed unpublished reagents/analytic tools; T.A.B. and M.H.D. analyzed data; T.A.B., M.H.D., and A.M.O. wrote the paper.

This work was supported by the Medical Research Council (U.1055.01.002.00007.01 and U.1055.01.002.00001.01) and by generous funding from the James S. McDonnell Foundation. T.A.B. was supported by an Alban European Union doctoral fellowship, a Consejo Nacional de Investigaciones Cientíicas y Técnicas fellowship, and Marie Curie Incoming International Fellowship Grant. We thank Christian Schwarzbauer for developing the ISSS sequence, the radiographers and staff at the Wolfson Brain Imaging Centre, University of Cambridge for their help with data acquisition, Jack Rogers for analysis of the behavioral data, and Matthew Brett for advice on image processing.

Correspondence should be addressed to Tristan A. Bekinschtein, Medical Research Council Cognition and Brain Sciences Unit, 15 Chaucer Road, CB2 7EF Cambridge, UK. E-mail: Tristan.bekinschtein@mrc-cbu.cam.ac.uk.

DOI:10.1523/JNEUROSCI.5058-10.2011

Copyright $\odot 2011$ the authors $\quad 0270-6474 / 11 / 319665-07 \$ 15.00 / 0$ because a single meaning of "funny" prevails. Both the IFG and left inferior temporal gyrus (ITG) have been shown to be activated in response to (nonhumorous) semantically ambiguous sentences (Rodd et al., 2005; Zempleni et al., 2007)—do these regions play a similar role in our ability to get a joke?

Many jokes do not use words with multiple meanings. "Why did Cleopatra bathe in milk? Because she couldn't find a cow tall enough for a shower!" has a similar structure as the joke about the cannibals, but does not depend on semantic ambiguity. This distinction between jokes that do and do not rely on ambiguity may map on to the incongruity resolution model of humor (Hempelmann and Ruch, 2005), with ambiguous jokes having unresolvable incongruity (both meanings of "funny" remain possible in the clown joke) and unambiguous jokes being more resolved (Cleopatra's shower). A critical test of this account concerns the impact of ambiguous words in stimuli that are not funny. Many common words in English have multiple meanings (Rodd et al., 2002), yet sentences containing ambiguous word are rarely funny (e.g., "what was the problem with the other coat? It was difficult to put on with the paint-roller."). Using these materials, we can assess whether common neural mechanisms are involved in ambiguity resolution in sentences and puns that depend on unresolvable ambiguity. By testing for interactions between humor and ambiguity resolution, we can assess mechanisms associated with ambiguity/incongruity resolution in puns.

In this study, we used fMRI to investigate the relationship between semantic ambiguity and humor using four types of tightly matched sentences that crossed two factors: the presence/ absence of humor (jokes vs non-jokes) and the presence/absence of ambiguous words. We predicted that jokes would activate a 
network of brain regions involving the IFG and the LITG, but only if they involved semantically ambiguous words. In addition, by comparing these items to semantically unambiguous jokes and nonjokes containing semantic ambiguity, we sought to reveal those aspects of getting a joke that are not dependent on semantic ambiguity and the extent to which conventional ambiguity resolution dissociates from humor processing.

\section{Materials and Methods}

Materials. There were five experimental conditions: (1) ambiguous sentences (AS) in which critical content words had multiple meanings, (2) matched unambiguous sentences (US), (3) ambiguous jokes (AJ), (4) low-ambiguity jokes (UJ), and (5) signal-correlated noise. Materials in the AS and US conditions were versions of those used by Rodd et al. (2010a) that were modified to follow the same set-up and punchline structure as the jokes, to have similar syntactic structures, and to be closely matched for the number of words and syllables. To generate disambiguations in the AS condition that were as surprising as those in the $\mathrm{AJ}$ condition, the sentences used contained an ambiguous word that was (typically) at the offset of the set-up line and was disambiguated with a low-frequency meaning at the end of the punchline (e.g., "What happened to the post? As usual, it was given to the best-qualified applicant." "Post" is more commonly a "wooden pole" or "mail delivery" rather than a "job"). Existing work has shown that such disambiguations are both unexpected (as quantified by sentence completion pretests) and give rise to elevated frontal-temporal activation (Rodd et al., 2005; Zempleni et al., 2007), yet are not funny.

One important difference between the AS and $\mathrm{AJ}$ conditions was that, in the $\mathrm{AJ}$ condition, the ambiguous word typically occurred at the end of the punchline and involved a paradoxical disambiguation in which both meanings were relevant (e.g., "Why were the teacher's eyes crossed? Because she could not control her pupils."). Ambiguous words in the AS and AJ conditions were either homonyms (both meanings have identical spelling, e.g., pupils) or homophones (the two meanings have the same pronunciation, but different spellings, e.g., mussel/muscle). The stimuli in all four conditions were recorded by a native English speaker using a lively prosody, rhythm, and intonation suitable for telling jokes. The duration of the individual sentences and jokes were matched across conditions and varied between $3.1 \mathrm{~s}$ to $7.5 \mathrm{~s}$.

A set of 23 jokes and sentences sampled approximately equally from the four conditions were converted into speech-spectrum signalcorrelated noise (Schroeder, 1968) using Praat software (http://www. praat.org). These stimuli have the same spectral profile and amplitude envelope as the original speech, but because all spectral detail is replaced with noise, they are entirely unintelligible. Although the amplitude envelope of speech (which is preserved in signal-correlated noise) can, in theory, provide cues to some forms of rhythmic and phonological information (Rosen, 1992), such cues are insufficient for the listener to recognize lexical items and extract any information about sentence meaning (Davis and Johnsrude, 2003).

Participants. Eighteen right-handed volunteers (nine females), aged 18 -35 were scanned; data from three participants were discarded due to Left posterior IFG.

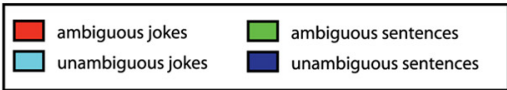

b

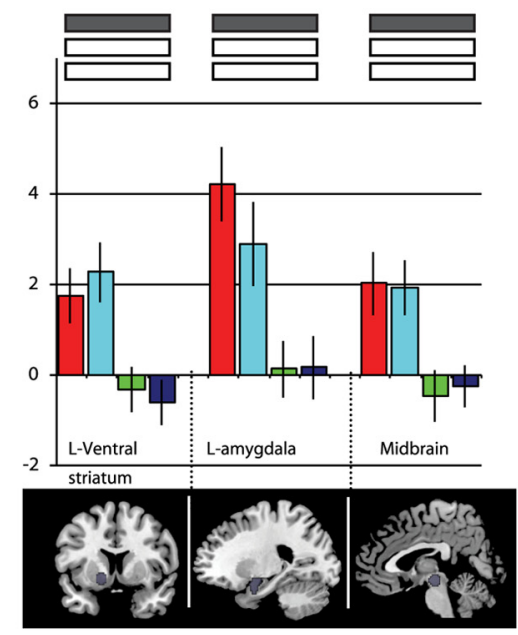

Figure 1. Results from the ROl analysis, calculated for the main effects (ambiguity and joke/non-joke) and interaction. $\boldsymbol{a}, \boldsymbol{b}$, Top, Bars show beta values (peak voxels for each of the stimuli conditions). $\boldsymbol{a}$, Bottom, Sagittal brain images showing the cortical Rols.
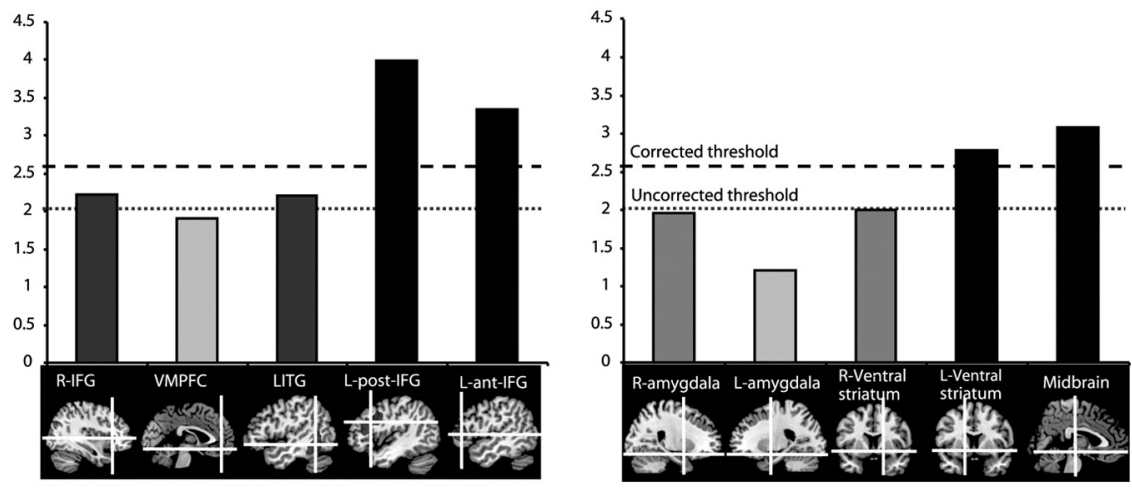

Figure 2. Top, Results from the focused ROl analysis, calculated for the funniness correlation. Bottom, Sagittal brain images showing the cortical ROIs (right) and the subcortical ROls (left). Left posterior IFG (L-post-IFG), left anterior IFG (L-ant-IFG), left ventral striatum, and midbrain ROls showed significant correlation with funniness ratings, corrected for multiple comparisons. Right IFG (R-IFG) and left ITG (LITG) were significantly correlated with funniness but at uncorrected levels.

excessive head movement and three participants' data were discarded due to scanner faults during data acquisition; the remaining 12 participants were included in the analysis. All participants were native speakers of English with no history of neurological illness, head injury, or hearing impairment. The study was approved by the Addenbrooke's Hospital Local Research Ethics Committee (Cambridge) and written informed consent was obtained from all participants.

Procedure. Volunteers were instructed to listen attentively to all of the stimuli and were advised that some of the sentences might be humorous but given no other task to do. An interleaved silent steady state (ISSS) imaging technique was used (Schwarzbauer et al., 2006). This sequence provides an optimal combination of high temporal resolution echoplanar imaging (EPI) data following a silent interscan interval such that there is minimal interference from concurrent scanner noise during stimulus presentation. The sequence also avoids T1-related signal decay during the acquisition of the EPI volumes by maintaining the steadystate longitudinal magnetization with a train of silent slice-selective excitation pulses during the (silent) period when auditory stimuli are 


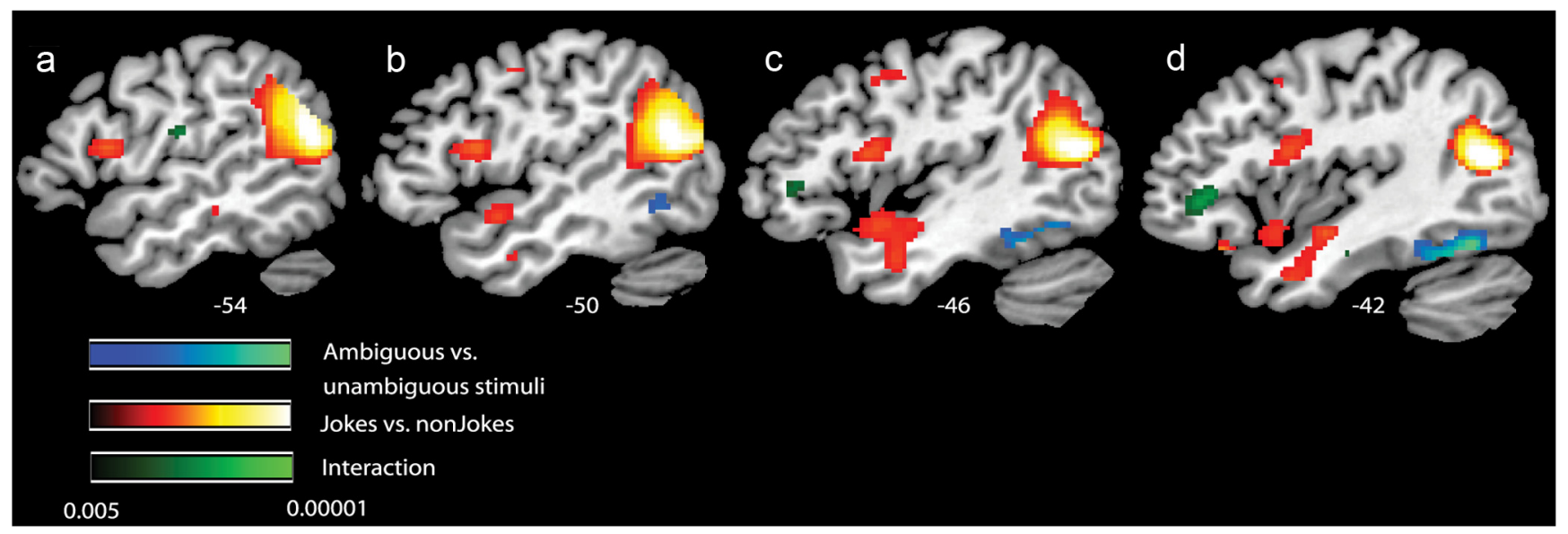

Figure 3. fMRI response for simple contrasts between jokes and non-jokes (red to yellow), ambiguous and unambiguous stimuli (blue to cyan), and the interaction (black to green). For display, contrasts are thresholded at $p<0.005$, uncorrected for whole-brain comparisons for at least 10 consecutive voxels. Activation maps are rendered onto a sagittal canonical T1 brain image.

presented. Volunteers heard a single sentence (or noise equivalent) in the $9 \mathrm{~s}$ silent period before a $12 \mathrm{~s}$ period of scanning. Stimulus presentation was timed so that the final word of the joke or sentence finished immediately before the onset of the sequence of scans. There were 23 trials of each stimulus type, including signal-correlated noise, and an additional 23 silent trials for the purpose of monitoring data quality (138 trials total). The experiment was divided into three sessions of 46 trials with a single silent trial added at the start of each scanning run to allow for the $\mathrm{T} 1$ signal to reach asymptote before stimulus presentation commenced. The order of stimulus items was pseudorandomized to ensure that the experimental conditions and rest scans were approximately evenly distributed among the three scanning sessions and that each condition occurred equally often after each of the other conditions. Session order was counterbalanced across participants. Stimuli were presented to both ears over high-quality headphones (Commander XG system; Resonance Technology) using DMDX software running on a Windows PC (Forster and Forster, 2003). To further attenuate scanner noise, participants wore insert earplugs.

Functional neuroimaging data were acquired using a Bruker Medspec 3T MR system with a head gradient set. Echo-planar image volumes were collected for each volunteer with a TR of $1.5 \mathrm{~s}, 8$ TRs following each of the 46 trials in each of the three 16 min scanning sessions. The silent interval between trials lasted $9 \mathrm{~s}$ (i.e., 6 TRs). The following imaging parameters were used: slice thickness, $4 \mathrm{~mm}$; interslice gap, $1 \mathrm{~mm}$; number of slices, 27; slice orientation, axial oblique; field of view, $24 \times 24 \mathrm{~cm}$, matrix size, $64 \times 64$, in-plane spatial resolution $3.75 \times 3.75 \times 4 \mathrm{~mm}$. The gradient ramp and plateau times for the ISSS sequence were repetition time of the gradient ramp $=3.0 \mathrm{~ms}$ and time of the plateau $=1.0 \mathrm{~ms}$ (for details, see Schwarzbauer et al., 2006). Acquisition was transverse-oblique, angled away from the eyes, and covered the whole brain.

The fMRI data were preprocessed and analyzed using Statistical Parametric Mapping software (SPM2, Wellcome Trust Centre for Neuroimaging, London, UK). Preprocessing steps included within-subject realignment, spatial normalization of the functional images to a standard EPI template and spatial smoothing using a Gaussian kernel of $12 \mathrm{~mm}$, suitable for random-effects analysis (Xiong et al., 2000). First-level statistical analysis was performed using a general linear model constructed and estimated for each participant. We used a finite impulse response (FIR) basis set, such that each of the eight scans that followed the stimuli in each of the five experimental conditions were separately averaged. Scans following silent interscan intervals were included in the model as an unmodeled, implicit baseline. Movement parameters from the realignment stage of the preprocessing and dummy variables coding the three separate scanning sessions were included as covariates of no interest. No high-pass filtering or correction for serial autocorrelation was used in estimating the least-mean-squares fit of single-subject models. Although this prevents accurate assessment of the statistical significance of activation differences in single participants (as no correction is made for temporal correlations between scans), this has no impact on the validity of random-effect group analyses. The mean activity level in each condition over time can be reliably computed for each participant tested, as unmodeled temporal autocorrelation has no impact on estimates of the mean effect, only on estimates of the degree of scan-to-scan variation (for further discussion, see Schwarzbauer et al., 2006). Our analysis procedure focuses on the significance of activation estimates across the group of participants tested using intersubject variation as a random effect. Following estimation, contrasts between conditions were computed using weights derived from the predicted magnitude of the BOLD response over the eight time points in the FIR model using the SPM canonical HRF. In a subsequent analysis, participant-specific parametric modulators were added to each of the joke and non-joke conditions to investigate the relationship between neural responses and the rated funniness of each of the (joke and non-joke) sentences.

Images containing weighted parameter estimates from each participant were entered into second-level group analyses in which intersubject variation was treated as a random effect (Friston et al., 1999). Given the hypotheses derived from previous work in this area, a region-of-interest (ROI) statistical analysis was performed (Poldrack et al., 2008). Using the MarsBar SPM toolbox (Brett et al., 2002), 10 ROIs were defined. Three of these were defined based on the results of the comparison between sentences containing ambiguous words and sentences with no ambiguous words reported previously by Rodd et al. (2005) — specifically, a left posterior inferior frontal region $\left(-45,19,25 ; 6584 \mathrm{~mm}^{3}\right)$, right inferior frontal region $\left(36,25,3 ; 800 \mathrm{~mm}^{3}\right)$, and left inferior temporal region $\left(-51,-49,-9 ; 6696 \mathrm{~mm}^{3}\right)$. Two further cortical ROIs were defined based on the results of an earlier study of verbal jokes (Goel and Dolan, 2001) and included the anterior IFG (pars triangularis; - 52, 36, 6; 4144 $\left.\mathrm{mm}^{3}\right)$ and the ventromedial prefrontal cortex $\left(0,45,-12 ; 3984 \mathrm{~mm}^{3}\right)$. In several previous studies of humor, ratings of funniness have been shown to correlate with activity in subcortical structures (amygdalae, ventral striatum, and midbrain), suggesting that these regions code for the reward that is often subjectively associated with getting the joke (Mobbs et al., 2003, 2005; Azim et al., 2005). Therefore in this study, the amygdalae were identified from the MNI ROI atlas (left/right: $-23 / 23$, $\left.-2 /-2,-19 /-19 ; 1760 \mathrm{~mm}^{3}\right)$, small spherical ROIs were created for the left/right ventral striatum $\left(-16 / 16,7 / 7,-7 /-7 ; 896 \mathrm{~mm}^{3}\right)$, and midbrain $\left(0,-20,-11 ; 896 \mathrm{~mm}^{3}\right)$. The ROI threshold was set at $p<0.05$, corrected for multiple comparisons. All ROIs selected are displayed in Figure 2.

After the scanning session, each participant was interviewed and asked to rate the funniness of every joke and non-joke sentence that had been presented during the scanning session on a 1 to 7 scale ( $1=$ not funny at all, 7 = very funny). 


\section{Results}

\section{Behavioral results}

The participants rated the joke stimuli significantly funnier than the non-joke sentences. Thus, a two-way repeated-measures ANOVA revealed a main effect of sentence type (jokes vs nonjokes; $\left.F_{(1,11)}=97.75, p<0.0001\right)$. The main effect of ambiguity was not significant $\left(F_{(1,11)}=0.51, p=0.49\right)$ and there was no significant interaction between ambiguity and sentence type $\left(F_{(1,11)}=3.02, p=0.11\right)$. Funniness ratings did not predict head movements during the scanning session $\left(R^{2}=0.12\right.$, not significant).

\section{fMRI results}

\section{Region of interest}

On the basis of previous work in this area, an ROI analysis was performed to characterize the different contributions of each sentence type in terms of semantic ambiguity and funniness. Ten ROIs were included, as described above. When all stimuli containing ambiguous words (both jokes and non-jokes) were compared with matched jokes/non-jokes without ambiguous words, significant activity was observed in the left ITG $(t=2.76 ; p=$ $0.009)$ and in the left anterior IFG $(t=2.03 ; p=0.033)$ (Fig. 1). The former results replicate the findings of Rodd et al. (2005), who reported significant ambiguity-related activity in the same region of the ITG. The latter result demonstrates that there is a significant ambiguity-related change in the region identified by Goel and Dolan (2001) as being involved in hearing verbal jokes. This region was anterior to the inferior frontal region previously reported by Rodd et al. (2005).

The jokes versus non-jokes contrast revealed much stronger and more widely spread activity in the jokes condition, with significant differences between conditions in all ROIs but higher activity in the amygdalae (left: $t=2.95, p=0.006$; right: $t=2.77$, $p=0.009)$ and midbrain $(t=3.03, p=0.006)$. The interaction between the joke/non-joke and ambiguity factors revealed significant activity only in the left anterior IFG ROI $(t=2.44, p=$ 0.016) (Fig. 1).

Ratings of funniness were correlated with activity in the 10 ROIs (Fig. 2). There were strong associations between BOLD signal change and funniness in the midbrain $(t=3.08, p=$ $0.005)$, the left ventral striatum $(t=2.78, p=0.008)$, and the left anterior $(t=3.99, p=0.001)$ and posterior $(t=3.34, p=$ $0.003)$ IFG.

\section{Whole-brain analysis}

A supplementary whole-brain analysis was also performed to examine whether the regions explored in the ROIs were the only ones active during the main contrasts and interactions (a liberal threshold was set to $p<0.001$, uncorrected, 10 voxels cluster). When jokes were compared with non-joke sentences, substantial activity (outside the ROIs) was observed in the temporoparietal junction from the angular gyrus bilaterally, spreading to the posterior superior and middle temporal gyri [Brodmann areas (BA) 39 and 21 ; left side, 1512 voxels; right side, 1682 voxels]. A significant increase in activity was also observed in the left inferior frontal gyrus (BA 6/44, 273 voxels) (Fig. 3, Table 1). Subcortically, the ventral tegmental area (VTA) was activated ( 929 voxels) (Fig. 4) and a cluster of activity was observed that included the left hypothalamus, amygdala, and ventral striatum (367 voxels) (Fig. 4 , Table 1). The reverse contrast between non-joke sentences and jokes revealed no significant areas of activity.

The other main contrast, between all ambiguous and nonambiguous sentences (including both jokes and non-jokes), revealed
Table 1. fMRI activations

\begin{tabular}{|c|c|c|c|c|c|}
\hline \multirow{2}{*}{$\mathrm{BA}$} & \multirow[b]{2}{*}{ Region } & \multicolumn{3}{|c|}{ Coordinates } & \multirow[b]{2}{*}{ Zscore } \\
\hline & & $x$ & $y$ & $z$ & \\
\hline \multicolumn{6}{|c|}{ Jokes versus non-jokes } \\
\hline \multirow[t]{2}{*}{$39-21$} & Upper L MTG & -42 & -66 & 18 & 6.64 \\
\hline & & -52 & -60 & 22 & 6.2 \\
\hline 39 & Upper R MTG & 48 & -58 & 18 & 4.98 \\
\hline \multirow[t]{6}{*}{$22-21$} & Posterior R MTG & 64 & -40 & 6 & 4.93 \\
\hline & & 62 & -28 & -6 & 4.64 \\
\hline & Midbrain & -4 & -30 & -2 & 4.8 \\
\hline & & 0 & -22 & -2 & 4.71 \\
\hline & & 6 & -30 & 0 & 4.64 \\
\hline & R amigdala/V Str. & 10 & 0 & -20 & 4.66 \\
\hline 23 & L posterior cingulum & 0 & -54 & 24 & 4.46 \\
\hline 19 & L upper occipital & -34 & -78 & 40 & 4.07 \\
\hline \multirow[t]{5}{*}{$38-21$} & R superior temporal pole & 54 & 6 & -12 & 3.85 \\
\hline & & 46 & 8 & -24 & 3.58 \\
\hline & L hippocampus/amygdala/V Str. & -16 & 0 & -26 & 3.75 \\
\hline & & -42 & -8 & -12 & 3.56 \\
\hline & & -48 & 2 & -12 & 3.56 \\
\hline \multirow[t]{2}{*}{$38-47$} & L orbitofrontal & -42 & 30 & -18 & 3.72 \\
\hline & & -34 & 26 & -14 & 3.27 \\
\hline 20 & R fusiform & 40 & -32 & -24 & 3.61 \\
\hline \multirow[t]{2}{*}{44} & L frontal operculum & -52 & 12 & 14 & 3.61 \\
\hline & & -40 & 4 & 22 & 3.54 \\
\hline 20 & L MTG & -58 & -26 & -12 & 3.61 \\
\hline \multirow{3}{*}{$\begin{array}{l}48 \\
20-30\end{array}$} & R IFG triangularis & 32 & 16 & 24 & 3.57 \\
\hline & L fusiform/L hippocampus & -32 & -24 & -28 & 3.52 \\
\hline & & -22 & -20 & -28 & 3.15 \\
\hline \multirow[t]{2}{*}{$32-10$} & L SFG/L anterior cingulum & 0 & 52 & 16 & 3.48 \\
\hline & & -4 & 48 & 2 & 3.35 \\
\hline \multirow[t]{2}{*}{22} & L MTG & -60 & -34 & 6 & 3.41 \\
\hline & Pons-midbrain junction & 0 & -26 & -24 & 3.38 \\
\hline 47-11 & Rorbitofrontal & 32 & 24 & -18 & 3.28 \\
\hline 24 & R anterior cingulum & 2 & 20 & 22 & 3.27 \\
\hline \multirow[t]{2}{*}{6} & L precentral & -50 & -2 & 46 & 3.27 \\
\hline & & -46 & 6 & 46 & 3.27 \\
\hline 6 & LSFG & -16 & 8 & 60 & 3.18 \\
\hline \multicolumn{6}{|c|}{ Ambiguous versus } \\
\hline \multicolumn{6}{|c|}{ unambiguous } \\
\hline \multicolumn{6}{|c|}{ sentences } \\
\hline 19 & Lfusiform & -42 & -62 & -16 & 5.23 \\
\hline 37 & LITG & -44 & -48 & -20 & 4.89 \\
\hline \multicolumn{6}{|c|}{ Unambiguous versus } \\
\hline \multicolumn{6}{|c|}{ ambiguous sentences } \\
\hline 39 & L ang/RMTG & -44 & -62 & 26 & 5.92 \\
\hline 21 & Upper R MTG & 50 & -52 & 14 & 5.84 \\
\hline \multicolumn{6}{|c|}{ Jokes/ambiguity interaction } \\
\hline \multirow[t]{3}{*}{$47-11$} & L orbitofrontal//FG triangularis & -24 & 42 & -2 & 4.87 \\
\hline & & -32 & 36 & 4 & 4.73 \\
\hline & Rhippocampus & 40 & -6 & -24 & 4.59 \\
\hline
\end{tabular}

Coordinates of activation foci together with $Z$ scores and an estimate of location relative to gross anatomy for each contrast of interest. SFG, Superior frontal gyrus; MTG, middle temporal gyrus; $V$ Str., ventral striatum; $R$, right; L, left. All peak voxels exceeding $p<0.001$ are reported (at least 10 voxels clusters). Each area may be composed of one or several clusters.

an area of activation in the left posterior temporal lobe (BA 37/20, 168 voxels) that included portions of the left fusiform and inferior temporal gyri (Fig. 3). The location of this peak of activity was very similar to that of a peak reported previously by Goel and Dolan (2001) during verbal jokes. This change in activity was also very similar to a peak observed previously by Rodd et al. (2005), when (non-joke) sentences containing ambiguous words were compared with sentences containing no ambiguous words. The reverse contrast, between nonambiguous and ambiguous stimuli, revealed clusters of activity in the temporoparietal junction (TPJ), centered in the left angular gyrus (BA 39, 599 voxels) and spreading through the superior temporal gyrus to the upper tip of 

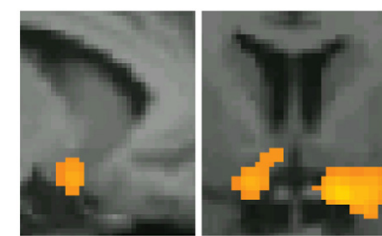

a

$$
\text { Ventral striatum }
$$

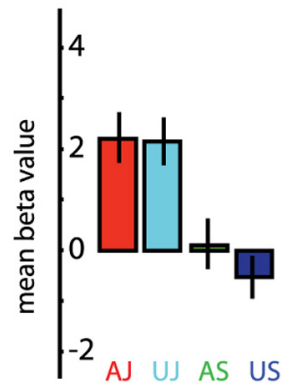

\section{ambiguous jokes}

unambiguous jokes

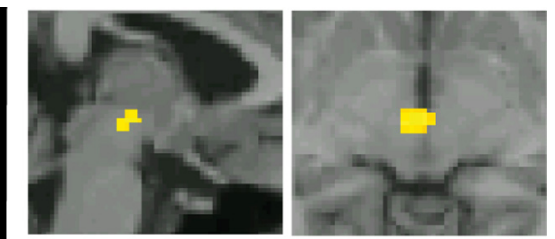

Midbrain (VTA)

b

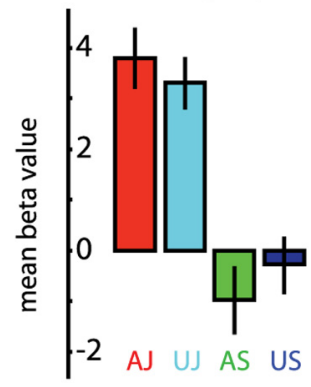

Figure 4. fMRI response for simple contrast between jokes and non-jokes. $\boldsymbol{a}$, For display, contrasts are thresholded at $p<0.001$ uncorrected for whole-brain comparisons. Activation maps are rendered onto canonical T1 brain images. Top left, Sagittal view of the left ventral striatum activation; Top right, coronal view of the ventral striatum bilaterally spreading to the right amygdala. $\boldsymbol{b}$, Top left, Sagittal views of midbrain activation; Top right, coronal views of midbrain activation. $\boldsymbol{a}, \boldsymbol{b}$, Bottom, Beta values (peak voxels for each of the stimuli conditions).

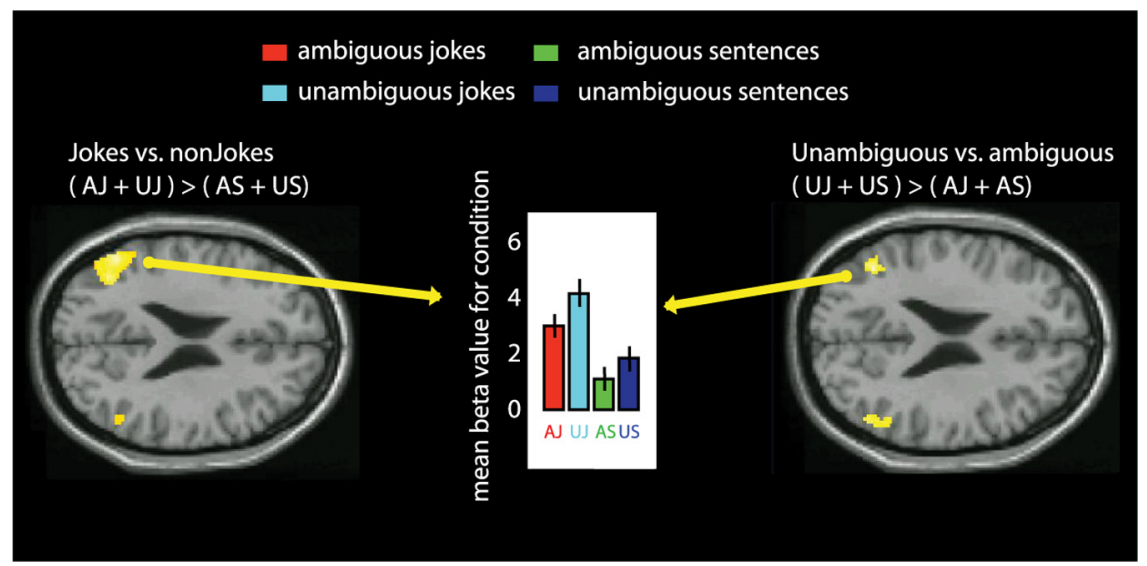

Figure 5. fMRI response for simple contrast between jokes versus non-jokes (left) and unambiguous stimuli versus ambiguous stimuli (right). Contrasts are thresholded at $p<0.001$ uncorrected for whole-brain comparisons and activation maps are rendered onto axial canonical T1 brain images. Left, Axial view; TPJ activity in the main jokes contrast (AJ + UJ) $>$ (AS + US). Right, Axial view; TPJ activity for unambiguous versus ambiguous stimuli (UJ + US) $>(A J+A S)$. Middle, Bar graph shows mean beta values for each condition, showing higher activity for the jokes over the non-jokes and higher activity for combined unambiguous sentences and jokes over ambiguous sentences and puns.

the right middle temporal gyrus (BA 21, 549 voxels) (Fig. 5, Table 1). This effect seems to be driven by both jokes and non-jokes; although there is a trend toward higher activity in the unambiguous jokes compared with the ambiguous jokes, this simple effect was not reliable. In the left and right TPJ, there are areas of common activity between the main contrast jokes versus non-jokes $(\mathrm{AJ}+\mathrm{UJ})>(\mathrm{AS}+\mathrm{US})$ and the reverse contrast unambiguous versus ambiguous stimuli (UJ + US) $>(A J+A S)$ (Fig. 5).

When the interaction between ambiguity and jokes was examined, a significant change in activity was observed in the left IFG orbitalis and triangularis, spreading to the middle frontal gyrus (MFG; BA 45/47, 658 voxels), which was specifically associated with ambiguous jokes (Table 1). No other significant effects were observed, nor did any brain areas show an interaction consistent with additional activity for unambiguous jokes.

BOLD activity correlated with funniness in the left IFG (anterior and posterior; BA 45,145 voxels) and the dorsolateral prefrontal cortex, bilaterally, including the precentral gyrus, with activity spreading to the IFG and MFG on the left and IFG pars opercularis on the right. In the parietal lobe, the response in the angular gyri were also correlated with the degree of funniness (BA 40/7; left, 294 voxels; right, 235 voxels).

\section{Discussion}

In this study, jokes that depend on semantically ambiguous words were compared with similar high-ambiguity sentences that were not funny, and with verbal jokes that did not depend on semantic ambiguity. The results confirm that both the left ITG and IFG are involved in resolving semantic ambiguities (Rodd et al., 2005, 2010b), while a more widespread network (that includes both regions) is involved in processing humorous jokes when compared with matched nonhumorous material. Hearing jokes was associated with increased activity in a network of subcortical regions, including the amygdalae, the ventral striatum, and the midbrain, which have been implicated in experiencing positive reward. Moreover, activity in these regions correlated with the subjective ratings of funniness of the material.

That effects of both humor and ambiguity are seen in a common frontotemporal network has important implications for neuroscientific theories of humor in general. Previous work has shown that language-dependent humor generates more IFG activity than matched visual humor (Watson et al., 2007). Here we show that these same inferior frontal regions respond more strongly to the processing of humorous material and even more for jokes that depend on semantic ambiguity. Clearly then, the role of these regions extends beyond those processes that are involved in perceiving and understanding semantically ambiguous words in sentences (Rodd et al., 2005; Zempleni et al., 2007).

While there exists an extensive psychological literature on the cognitive processes that underpin humor, relatively little has been done to relate these processes to specific brain regions (Gallagher et al., 2000; Goel and Dolan, 2001; Mobbs et al., 2003, 2005; Moran et al., 2004; Azim et al., 2005; Marjoram et al., 2006; Wild et al., 2006; Samson et al., 2009). One framework, the incongruity-resolution model (Suls, 1972; Ruch, 1992), considers humor to be a problem-solving task, where incongruity between the punch line and the set-up line must be solved by a 
change of frame of reference, or a shift of context. Ruch and colleagues (Ruch, 1992; Wild et al., 2003, 2006) propose three aspects of humor: incongruity-resolution, nonsense, and sexual humor (Hempelmann and Ruch, 2005). The last is primarily related to joke content while the other two refer to a two-stage process of understanding humor. This states that there is first a discovery of incongruity and second a stage where it resolves, partially resolves, or creates new absurdities or incongruities. Similarly, the coherence account (Coulson, 2001) proposes a frame-shifting step that involves the comparison of information in working memory with preexisting long-term knowledge. Within either framework, the current study allows the purely semantic aspects of jokes to be disentangled from surprise or reestablishment of coherence. We included controlled forms of semantic ambiguity in humorous (jokes) and nonhumorous (non-jokes) contexts. In both cases, elevated frontotemporal activity was observed for ambiguous relative to matched control conditions, but an additional response specific to ambiguous jokes was also seen in the left anterior IFG, confirmed by a significant interaction between ambiguity and humor.

Our study allows, therefore, a more precise account of how IFG involvement in incongruity resolution contributes to the appreciation of jokes that depend on semantic ambiguity. It is not the frame shift or resolution of incongruity that creates humor (since this is present in non-joke semantic ambiguity resolution), but rather a continuing process of ambiguity resolution that is unique to the ambiguous jokes. A critical difference is that in puns such as "Why don't cannibals eat clowns? Because they taste funny!", the critical word (funny) activates multiple meanings (e.g., odd/bad and amusing). Crucially, both possible meanings are relevant and remain plausible given the overall meaning of the punchline (Alexander, 1997). In the context of the incongruityresolution model (Hempelmann and Ruch, 2005), these stimuli are moderately incongruous since there is no resolution of the incongruity created by the semantic ambiguity as both meanings are possible. This is unlike the process that occurs during comprehension of sentences containing ambiguous words in which one or the other meaning is ultimately selected (e.g., "What was the problem with the other coat? It was difficult to put on with the paint roller"; "coat" here only refers to a layer of paint once the ambiguity has been resolved, and can no longer refer to a garment). The results of the current study reveal increased anterior IFG activity associated with paradoxical disambiguations in which both meanings remain plausible, as in humorous puns, compared to conventional disambiguation of ambiguous words and to jokes that do not depend on ambiguity.

The analysis of the neural bases of the incongruity-resolution model have been explicitly tested in a series of studies (Wild et al., 2006; Samson et al., 2008, 2009) showing the temporoparietal junction as a key area in the detection and processing of incongruity. Consistent with this finding, we observed left and right TPJ activated by jokes versus non-jokes. However, our results also showed that these areas were more strongly activated by unambiguous than ambiguous stimuli regardless of humor, without any significant interaction. Thus, our study did not provide evidence for increased TPJ activity during incongruity-resolution humor versus nonsense humor (Samson et al., 2009).

The results of the main comparisons also provide evidence concerning the role of the left ITG in semantic ambiguity resolution. We show that the LITG participates in both semantic aspects of language comprehension (such as resolution of ambiguity) and processing of the bistable meanings in puns. But to success- fully process the set changes in the joke stimuli, the network recruited is broader, involving a frontoparietal network as found in other executive processes (Naccache and Dehaene, 2001; Duncan and Owen, 2000).

The cognitive processes underlying the resolution of verbal humor seem to be part of executive functions (set shifting, reestablishment of context). These functions have been largely related to conscious processing in behavioral and functional imaging studies (Posner and Rothbart, 1998; Koch and Tsuchiya, 2007) and it has been suggested that this pattern of activation is a prerequisite for conscious processing (Dehaene and Changeux, 2011). The psychology of mirth suggests that the understanding of a funny situation requires awareness (Attardo, 2001), and the cortical activation associated with getting the joke in this study suggests that the frontoparietal network is a neural correlate of the specific conscious process of mirth.

Some of the brain regions that were activated in the comparison between jokes and non-jokes are related to the reward associated with getting a joke (Azim et al., 2005). Previous investigations have suggested that various subcortical structures (e.g., amygdala, nucleus accumbens, ventral tegmental area, hypothalamus) contribute to this aspect of humor appreciation (Mobbs et al., 2003, 2005; Moran et al., 2004, Schwartz et al., 2008), as opposed to frontal and posterior temporal regions, which are assumed to be more involved in humor processing. In our study, it was the midbrain, the left ventral striatum, and left IFG that correlated most highly with subjective ratings of funniness (an estimate of the pleasure caused by the jokes), suggesting that the dopaminergic reward system is coding the funniness (and pleasure). Both the ventral striatum and the VTA reflect positive reward prediction errors (O'Doherty et al., 2006; D'Ardenne et al., 2008) and have been identified as relays in the reward system, which are active in self-reported happiness and monetary rewards (Knutson et al., 2001) during the detection of attractive faces (Cloutier et al., 2008) and in addiction (Reuter et al., 2005). Other possible interpretations suggest that part of the subcortical network activated could be due to feelings of exhilaration and/or motor/autonomous reaction such as smiling and changes in heart rate (Wild et al., 2003). Since we did not monitor autonomic reactions or face muscle activity, we cannot rule out these explanations. Nevertheless, the correlation between funniness and midbrain, the left ventral striatum and left IFG, and the lack of correlation between funniness and movement parameters during scanning suggest that the reward system was involved. A combined study of brain imaging and autonomic and facial muscle monitoring may help to disentangle these different contributions.

Clearly, more work is needed to fully dissect the neural and cognitive bases of the many different aspects and sources of humor that we encounter in our daily lives. The work presented here takes a step in this direction by showing neural continuity between the mechanisms involved in selecting the appropriate single meaning of ambiguous words in non-joke sentences and the humor created by selecting both meanings of an ambiguous word in puns. The association that was observed between the presence of double meanings in puns and activity in anterior regions of the left IFG associated with semantic selection processes illustrates one way in which humor builds on neurocognitive mechanisms for routine aspects of language comprehension. It will be for future work to find similar neural homologies in the mechanisms that underpin the other social and cognitive processes that evoke humor. 


\section{References}

Alexander RJ (1997) Aspects of verbal humour in English. Tubingen, Germany: Narr Franke Attempto Verlag.

Attardo S (2001) Humorous texts: a semantic and pragmatic analysis. Berlin: Mouton de Gruyter.

Azim E, Mobbs D, Jo B, Menon V, Reiss AL (2005) Sex differences in brain activation elicited by humor. Proc Natl Acad Sci U S A 102:16496-16501.

Brett M, Anton JL, Valabregue R, Poline JB (2002) Region of interest analysis using an SPM toolbox. Paper presented at 8th International Conference on Functional Mapping of the Human Brain, Sendai, Japan, June.

Cloutier J, Heatherton TF, Whalen PJ, Kelley WM (2008) Are attractive people rewarding? Sex differences in the neural substrates of facial attractiveness. J Cogn Neurosci 20:941-951.

Coulson S, (2001) Semantic leaps: frame-shifting and conceptual blending in meaning construction. Cambridge: Cambridge UP.

D’Ardenne K, McClure SM, Nystrom LE, Cohen JD (2008) BOLD responses reflecting dopaminergic signals in the human ventral tegmental area. Science 319:1264-1267.

Davis MH, Johnsrude IS (2003) Hierarchical processing in spoken language comprehension. J Neurosci 23:3423-3431.

Dehaene S, Changeux JP (2011) Experimental and theoretical approaches to conscious processing. Neuron 70:200-227.

Duncan J, Owen AM (2000) Common regions of the human frontal lobe recruited by diverse cognitive demands. Trends Neurosci 23:475-483.

Forster KI, Forster JC (2003) DMDX: a windows display program with millisecond accuracy. Behav Res Methods Instrum Comput 35:116-124.

Friston KJ, Holmes AP, Price CJ, Büchel C, Worsley KJ (1999) Multisubject fMRI studies and conjunction analyses. Neuroimage 10:385-396.

Gallagher HL, Happé F, Brunswick N, Fletcher PC, Frith U, Frith CD (2000) Reading the mind in cartoons and stories: an fMRI study of 'theory of mind' in verbal and nonverbal tasks. Neuropsychologia 38:11-21.

Goel V, Dolan RJ (2001) The functional anatomy of humor: segregating cognitive and affective components. Nat Neurosci 4:237-238.

Hempelmann CF, Ruch W (2005) 3 WD meets GTVH: breaking the ground for interdisciplinary humor research. Humour 18:353-388.

Knutson B, Adams CM, Fong GW, Hommer D (2001) Anticipation of increasing monetary reward selectively recruits nucleus accumbens. J Neurosci 21:RC159.

Koch C, Tsuchiya N (2007) Attention and consciousness: two distinct brain processes. Trends Cogn Sci 11:16-22.

Marjoram D, Job DE, Whalley HC, Gountouna VE, McIntosh AM, Simonotto E, Cunningham-Owens D, Johnstone EC, Lawrie S (2006) A visual joke fMRI investigation into theory of mind and enhanced risk of schizophrenia. Neuroimage 31:1850-1858.

Mobbs D, Greicius MD, Abdel-Azim E, Menon V, Reiss AL (2003) Humor modulates the mesolimbic reward centers. Neuron 40:1041-1048.

Mobbs D, Hagan CC, Azim E, Menon V, Reiss AL (2005) Personality predicts activity in reward and emotional regions associated with humor. Proc Natl Acad Sci U S A 102:16502-16506.

Moran JM, Wig GS, Adams RB Jr, Janata P, Kelley WM (2004) Neural correlates of humor detection and appreciation. Neuroimage 21:1055-1060.

Naccache L, Dehaene S (2001) Unconscious semantic priming extends to novel unseen stimuli. Cognition 80:215-229.

O’Doherty JP, Buchanan TW, Seymour B, Dolan RJ (2006) Predictive neu- ral coding of reward preference involves dissociable responses in human ventral midbrain and ventral striatum. Neuron 49:157-166.

Poldrack RA, Fletcher PC, Henson RN, Worsley KJ, Brett M, Nichols TE (2008) Guidelines for reporting an fMRI study. Neuroimage 40:409-414.

Posner MI, Rothbart MK (1998) Attention, self-regulation and consciousness. Philos Trans R Soc Lond B Biol Sci 353:1915-1927.

Reuter J, Raedler T, Rose M, Hand I, Gläscher J, Büchel C (2005) Pathological gambling is linked to reduced activation of the mesolimbic reward system. Nat Neurosci 8:147-148.

Rodd JM, Gaskell MG, Marslen-Wilson WD (2002) Making sense of semantic ambiguity: semantic competition in lexical access. J Mem Lang 46:245-266.

Rodd JM, Davis MH, Johnsrude IS (2005) The neural mechanisms of speech comprehension: fMRI studies of semantic ambiguity. Cereb Cortex 15:1261-1269.

Rodd JM, Johnsrude IS, Davis MH (2010a) The role of domain-general frontal systems in language comprehension: evidence from dual-task interference and semantic ambiguity. Brain Lang 115:182-188.

Rodd JM, Longe OA, Randall B, Tyler LK (2010b) The functional organisation of the front-temporal language system: evidence from syntactic and semantic ambiguity. Neuropsychologia 48:1324-1335.

Rosen S (1992) Temporal information in speech: acoustic, auditory and linguistic aspects. Philos Trans R Soc Lond B Biol Sci 336:367-373.

Ruch W (1992) Assessment of appreciation of humor: studies with the 3WD humor test. In Advances in personality assessment (Butcher JN, Spielberger CD, eds), pp 27-75. Hillsdale, NJ: Erlbaum.

Samson AC, Zysset S, Huber O (2008) Cognitive humor processing: different logical mechanisms in nonverbal cartoons: an fMRI study. Soc Neurosci 3:125-140.

Samson AC, Hempelmann CF, Huber O, Zysset S (2009) Neural substrates of incongruity-resolution and nonsense humor. Neuropsychologia 47:1023-1033.

Schroeder MR (1968) Reference signal for signal quality studies. J Acoust Soc Am 44:1735-1736.

Schwartz S, Ponz A, Poryazova R, Werth E, Boesiger P, Khatami R, Bassetti CL (2008) Abnormal activity in hypothalamus and amygdala during humour processing in human narcolepsy with cataplexy. Brain 131:514-522.

Schwarzbauer C, Davis MH, Rodd JM, Johnsrude I (2006) Interleaved silent steady state (ISSS) imaging: a new sparse imaging method applied to auditory fMRI. Neuroimage 29:774-782.

Suls J (1972) A two stage model for the appreciation of jokes and cartoons. In: Psychology of humor (Goldstein J, McGhee P, eds). New York: Academic.

Watson KK, Matthews BJ, Allman JM (2007) Brain activation during sight gags and language-dependent humor. Cereb Cortex 17:314-324.

Wild B, Rodden FA, Grodd W, Ruch W (2003) Neural correlates of laughter and humour. Brain 126:2121-2138.

Wild B, Rodden FA, Rapp A, Erb M, Grodd W, Ruch W (2006) Humor and smiling: cortical regions selective for cognitive, affective and volitional components. Neurology 66:887-893.

Xiong J, Rao S, Jerabek P, Zamarripa F, Woldorff M, Lancaster J, Fox PT (2000) Intersubject variability in cortical activations during a complex language task. Neuroimage 12:326-339.

Zempleni MZ, Renken R, Hoeks JC, Hoogduin JM, Stowe LA (2007) Semantic ambiguity processing in sentence context: evidence from eventrelated fMRI. Neuroimage 34:1270-1279. 